INPLASY

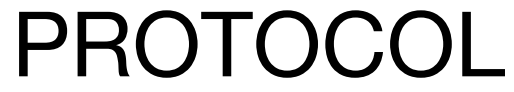

To cite: Liao et al. The predictive coefficient of obesity and anti-Mullerian hormone for ovarian reservation. Inplasy protocol 202090090. doi: 10.37766/inplasy2020.9.0090

Received: 25 September 2020

Published: 25 September 2020

Corresponding author:

Tuo Li

zoe_leeto@hotmail.com

Author Affiliation:

Shanghai Changzheng

Hospital

Support: RpNHC (QA2019037).

Review Stage at time of this submission: The review has not yet started.

Conflicts of interest:

The authors declare no conflict of interest.

\section{The predictive coefficient of obesity and anti-Mullerian hormone for ovarian reservation}

\author{
Liao, X1; Li, T2; Wang, J33.
}

Review question / Objective: Is there a correlation between obesity and ovarian reserve function in women of childbearing age (16-45 years old)?

Condition being studied: At present, we have established the research objective. Obesity is increasingly becoming a global health challenge. Nearly $\mathbf{5 0}$ per cent of women of childbearing age are overweight and obese, according to an American study. What's worse, obesity has been shown to be associated with decreased fertility in women. Whether obesity further affects reproductive health by affecting women's ovarian function has been controversial. Our research hopes to find a breakthrough in this problem and provide more powerful evidence by using meta-analysis. Our team investigated the current situation of the effect of the rising body mass index (BMI) on serum AMH levels in women. At present, no research has been found to conflict with us. Our team's medical workers have learned from some clinical experience that obesity poses a risk to women's fertility. We reviewed the literature on anovulation, uterine bleeding, and poor pregnancy outcomes (preterm birth, miscarriage or ectopic pregnancy) in obese women. We are also actively collecting background information on ovarian reserve function which can reflect reproductive function.

INPLASY registration number: This protocol was registered with the International Platform of Registered Systematic Review and Meta-Analysis Protocols (INPLASY) on 25 September 2020 and was last updated on 25 September 2020 (registration number INPLASY202090090).

\section{INTRODUCTION}

Review question / Objective: Is there a correlation between obesity and ovarian reserve function in women of childbearing age (16-45 years old)?

Rationale: The framework of this project is inspired by the work of some(Jia D et al, 
Thota $P$ et al, Pan $H$ et al.)research conducted in 2014-2018. Their work consisted of several variables and their effect on female infertility. Our aim to conduct the research is to verify the effect of obesity on female ovarian reserve diminishment and infertility, and culture on the work and correlational performance of two parameters (BMI and $\mathrm{AMH}$ ) to predict the progress of ovarian reserve in obese women and further potential risk on infertility.

Condition being studied: At present, we have established the research objective. Obesity is increasingly becoming a global health challenge. Nearly 50 per cent of women of childbearing age are overweight and obese, according to an American study. What's worse, obesity has been shown to be associated with decreased fertility in women. Whether obesity further affects reproductive health by affecting women's ovarian function has been controversial. Our research hopes to find a breakthrough in this problem and provide more powerful evidence by using metaanalysis. Our team investigated the current situation of the effect of the rising body mass index (BMI) on serum AMH levels in women. At present, no research has been found to conflict with us. Our team's medical workers have learned from some clinical experience that obesity poses a risk to women's fertility. We reviewed the literature on anovulation, uterine bleeding, and poor pregnancy outcomes (preterm birth, miscarriage or ectopic pregnancy) in obese women. We are also actively collecting background information on ovarian reserve function which can reflect reproductive function.

\section{METHODS}

Search strategy: We will search the electronic databases such as PubMed, EMBASE, Medline, and Wiley Online Library. Search strategy mainly included BMI, referring to the obesity standards set by WHO, and ovarian reserve marker, combined with medical subject title (MeSH) descriptor and text words.
Participant or population: Reproductive women age group (16-45 years).

Intervention: The studies we will includ are mainly cross-sectional studies (cohort studies and case-control studies). The exposure factors were overweight and obesity. In the study, we defined BMI > 25 $\mathrm{kg} / \mathrm{m} 2$ as overweight and $\mathrm{BMI}>30 \mathrm{~kg} / \mathrm{m} 2$ as obesity.

Comparator: We will use normal BMI (BMI < $25 \mathrm{~kg} / \mathrm{m} 2$ ) as control group, BMI > $25 \mathrm{~kg} /$ $\mathrm{m} 2$ as overweight group, BMI > $30 \mathrm{~kg} / \mathrm{m} 2$ as obesity group.

Study designs to be included: Observational studies, prospective cohort study, retrospective cohort study, casecontrol study or randomized controlled trials (RCTs).

Eligibility criteria: According to the research question (PICO), studies were included, if they met the following criteria: • Clinical studies assessing an independent association between obesity or overweight (calculated using BMI values) and ovarian reserve function; - Serum AMH levels were measured in all the study participants belonging to a reproductive age group (16-45 years); - Observational studies or randomized controlled trials (RCTs); • Only studies that represented the data in terms of mean and standard deviation and reported Pearson or Spearman correlation coefficients between serum AMH levels and BMI were selected. Thus, studies involving animals, human males, women not belonging to the reproductive age group were excluded, as were studies involving pregnant and lactating women. Moreover, studies involving medical or surgical treatment, and combination of severe complications (cancer, AIDS, etc.) were excluded. Literature search included studies published up to now and there was no restriction on language.

Information sources: We will search the electronic databases such as PubMed, EMBASE, Medline, and Wiley Online Library. Search strategy mainly included BMI, referring to the obesity standards set 
by WHO, and ovarian reserve marker, combined with medical subject title (MeSH) descriptor and text words. Search terms for ovarian reserve (MeSH: ovarian reserve, ovarian function), obesity (MeSH: obesity, obese, overweight, adiposity, polysarcia, weight measurement, fat weight, weight), and keywords such as 'anti-Mullerian hormone', 'AMH', 'Mullerian inhibitor', and 'body mass index' were used. 'BMI' was combined with a search filter for studies involving only human participants.

Main outcome(s): 1.Determine whether the mean serum AMH levels between obese and non-obese women have statistic difference; 2.Determine the transformed correlation coefficient between BMI and serum AMH levels, whether there a significant difference between obese and non-obese.

Additional outcome(s): Divided women into fertile, infertile and PCOS group, and test whether the serum AMH levels between obese and non-obese women have statistic difference in each group.

Data management: The $\mathbf{R}$ language (version 3.5.3.) will use to synthesize the effect sizes of the 16 selected studies. The mean serum AMH levels in obese women will be compared with non-obese women, and this will be performed using the random effect model to estimate the standardized mean difference. Beyond that, when analyzing the strength of a linear relationship between $B M I$ and ovarian reserve marker (AMH), the correlation coefficient will be meta-analyzed by calculating Fisher's r-to-z transformation (a variance stabilizing transformation for correlation coefficient).

Quality assessment / Risk of bias analysis: We will use STROBE criteria to quantify the quality of observational studies included in this meta-analysis. The biggest potential source of type I error (an increase of falsepositive results) in our meta-analysis is probably publication bias. Due to the clinical literature, statistically significant "positive" results have either a better chance of being published, are published earlier or in journals with higher impact factors, and are more likely to be cited by others. We will use the funnel plot to evaluate the presence of publication bias.

Strategy of data synthesis: We tend to use Random effects model in this metaanalysis. Because we assuming the true intervention effect of each study comes from a larger population, the confidence interval of the conclusion of the random effect model is large. At the same time, we use the $I^{2}$ value to determine the use of the model. When $I^{2}>50 \%$, there is the heterogeneity, we use random effects model, $\mathrm{I}^{2} \leq 50 \%$, use fixed effects model. When heterogeneity exists, sensitivity analysis and subgroup analysis will be used to explore the source of heterogeneity.

Subgroup analysis: When heterogeneity exists in the selected articles, subgroup analysis will be used to explore the sources of heterogeneity. Divided women into fertile, infertile and PCOS group, and test whether the serum AMH levels between obese and non-obese women have statistic difference in each group.

Sensibility analysis: Check potential outliers and influential research data in meta-analysis data by using leave-one-out estimates of the amount of sensitivity analyses for fixed/random-effects models. Furthermore, Cook's distances, DFFITS values, hat values, externally standardized residuals, covariance ratios and weights methods are also will be concluded.

Language: No.

Country(ies) involved: China.

Keywords: body mass index, BMI; antiMullerian hormone, AMH; ovarian reserve function; polycystic ovary syndrome, PCOS.

Contributions of each author:

Author 1 - XIAOXIAN LIAO.

Author 2 - TUO LI.

Author 3 - JIAWEI WANG. 